\title{
Pathologically confirmed breast cancer in Malawi: a descriptive study: Clinical profile of breast cancer
}

\section{R E. Kohler ${ }^{1}$, A Moses ${ }^{2}$, R Krysiak, N. G Liomba², S Gopal ${ }^{2}$}

1. Department of Health Policy and Management University of Chapel Hill at North Carolina

2. University of North Carolina Project Malawi

Correspondance: Racquel E. Kohler, Email: rkohler@email.unc.edu

\section{Abstract}

\section{Background}

Breast cancer is the most common female cancer in Africa, yet no published studies have investigated breast cancer in Malawi. Understanding the clinical profile of breast cancer is important to develop early diagnosis efforts.

\section{Aim}

To describe clinical and pathological characteristics of breast specimens from a pathology laboratory at a national teaching hospital.

\section{Methods}

Secondary analysis of pathology reports from July 2011 to September 2013.

\section{Results}

Among 85 breast cancer cases, 55\% were $<50$ years. Median tumor size was $4 \mathrm{~cm}$ and $49 \%$ were grade 3 . Median symptom duration was eight months.

\section{Conclusions}

Malawian women with breast cancer commonly have long symptom durations prior to diagnosis, young age, and poorly differentiated tumors. Improved clinical and pathological characterization, including hormone receptor status, are urgently needed to better understand this disease in Malawi.

\section{Introduction}

Breast cancer is the most common cancer and the leading cause of cancer death in Africa ${ }^{1}$. The breast cancer burden has increased across the continent with significant geographic and socioeconomic variation ${ }^{2}$. Low cancer awareness and lack of screening or control programs lead to many women being diagnosed late ${ }^{3}$. Although most breast cancer studies in Africa are small, retrospective descriptions with limited follow-up, available data suggest that African women are frequently diagnosed with advanced disease and have poor clinical outcomes. Some studies in African settings have shown high frequencies of aggressive tumour types with poorly differentiated tumours that are often hormone receptor (HR) negative. However, HR status assessment is not routinely done in many countries including Malawi ${ }^{4-6}$. This is partly due to an extreme scarcity of diagnostic pathology services in Africa ${ }^{7}$. In Malawi's Central Region, pathology diagnostic services were bolstered by the establishment, in July 2011, of a diagnostic pathology laboratory at Kamuzu Central Hospital $(\mathrm{KCH})$, a teaching hospital in Lilongwe ${ }^{8}$. At $\mathrm{KCH}$, efforts are ongoing to routinely assess $\mathrm{HR}$ status for all confirmed breast cancers, leveraging clinical research collaborations which provide immunohistochemistry reagents and technical support for new staining procedures ${ }^{8}$. This institutional database provides important data for understanding breast cancer in the Malawian context.

Breast cancer accounts for $8 \%$ of female cancers in Malawi ${ }^{9,10}$.
In a previous report from a histopathology laboratory in the Southern Region of the country, breast biopsies were the second most common type of biopsies sent to the laboratory, after esophageal specimens ${ }^{11}$. Understanding the clinical spectrum of breast cancer in Malawi is important to develop early diagnosis efforts to address high mortality rates. This cross-sectional study aimed to describe the pathological characteristics of breast specimens submitted to the KCH Pathology Laboratory, along with the clinical features corresponding to these specimens.

\section{Methods}

We reviewed KCH pathology reports from July 2011 to September 2013. KCH is a tertiary referral hospital for the Central Region of Malawi and the only hospital with pathology services in this region. Suspected breast cancer may be diagnosed after a core biopsy, fine needle aspiration (FNA), excisional or incisional biopsy, or surgical specimen. The KCH pathology database includes information on age, sex, HIV status, nature of the specimen, date of collection, date of receipt, date of report, clinical notes, macroscopy, microscopy, and diagnostic conclusion. For confirmed breast cancers, we abstracted data on tumor size, grade, lymph node involvement, and symptoms when available. HR status was not routinely available during this period and clinical data from patient charts were unavailable for review. To minimize errors during data abstraction, two co-authors independently reviewed reports of all specimens and discussed discrepant results to reach consensus. We excluded specimens from men and inadequate or non-representative specimens. We used descriptive statistics to report proportions and frequencies assessed, and used Pearson's Chi-square and t-tests to determine statistical significance. All analyses were performed using Stata version 12. This study was reviewed by the Malawian National Health Sciences Review Committee and exempted of patient informed consent due to the nature of secondary analyses of routinely collected data; it was also approved by the University of North Carolina institutional review board.

\section{Results}

There were 238 breast specimens in the pathology database during the 26-month study period. Eleven specimens from men (five were cancer) and 12 non-diagnostic specimens were excluded. We included one specimen per woman using the largest submitted specimen as the definitive report, leaving 193 women as shown in Table 1. The age range of patients was $12-89$ with a median of 34 years. Fifty-five percent (106) of the reports had a non-cancer diagnosis; two women were diagnosed with lymphoma. Fibroadenoma made up $87 \%$ of the benign breast diagnoses. Women without cancer were younger than those with cancer (median age 24 vs 46 years, $\mathrm{p}<0.001)$.Characteristics of the 85 confirmed breast cancer cases are displayed in Table 2. Fifty-five percent of cases occurred in women $<50$ years old. Invasive ductal carcinoma was the most common histologic type (86\%). Histologic grade was available for 53 cases and $49 \%$ were grade 3 . Of 42 specimens with lymph nodes submitted to the lab, $83 \%$ had involvement by cancer. Among women with invasive cancer, two had Paget's disease and four women had ductal 
carcinoma in situ. Clinical notes were available for 44 women. Although the notes indicated a palpable breast mass as the only symptom for 13 cases, many others included multiple symptoms. Commonly reported symptoms included fixed or mobile axillary lymphadenopathy (17), ulcerated lesion or mass (9), breast enlargement/swelling (5), peau d'orange (3), breast pain (3), multiple breast masses (2), nipple discharge (2), and nipple retraction (2). Of these, 30 had notes on symptom duration; the median reported length was 8 months (range 2-24 months) and $47 \%$ had symptoms for $>12$ months. Median tumor size was $4 \mathrm{~cm}$ (range 1-10 cm) and $45 \%$ were $\geq 5 \mathrm{~cm}$. TNM stage could not be assigned retrospectively for most confirmed cases using available data.

Table 1. Descriptive summary of breast biopsy reports at Kamuzu Central Hospital in Lilongwe, Malawi

\begin{tabular}{|c|c|c|c|c|}
\hline & $\begin{array}{c}\text { Breast } \\
\text { Cancer } \\
85(44 \%)\end{array}$ & $\begin{array}{c}\text { No Breast } \\
\text { Cancer } \\
108(56 \%)\end{array}$ & $\begin{array}{l}\text { Total } \\
193 \\
\end{array}$ & $p$-value \\
\hline Age group & & & & $<0.001$ \\
\hline$<30$ & $9(10.6)$ & $75(69.4)$ & $84(43.5)$ & \\
\hline 30-39 & $18(21.2)$ & $19(17.6)$ & $37(19.2)$ & \\
\hline $40-49$ & $20(23.5)$ & $9(8.3)$ & $29(15.0)$ & \\
\hline $50-59$ & $13(15.3)$ & $4(3.7)$ & $17(8.8)$ & \\
\hline 260 & $25(29.4)$ & $1(1.0)$ & $26(13.5)$ & \\
\hline \multicolumn{5}{|l|}{ Specimen type } \\
\hline FNA & $17(20.0)$ & $23(21.3)$ & $40(20.7)$ & \\
\hline Tissue biopsy & $27(31.8)$ & $36(33.3)$ & $63(32.6)$ & \\
\hline Tumor mass & $41(48.2)$ & $49(45.4)$ & $90(46.6)$ & \\
\hline
\end{tabular}

Table 2. Clinical characteristics of confirmed breast cancer cases at Kamuzu Central Hospital in Lilongwe, Malawi

\begin{tabular}{lccc}
\hline & $<50$ years old & 250 years old & Total \\
& $47(55.3 \%)$ & $38(44.7 \%)$ & $n=85$ \\
\hline Histologic Type & & & \\
Ductal & $31(66.0)$ & $25(65.8)$ & $56(65.9)$ \\
Lobular & $6(12.8)$ & $3(7.9)$ & $9(10.6)$ \\
Missing & $10(21.3)$ & $10(26.3)$ & $20(23.5)$ \\
Tumor Size & & & \\
T1 & $3(6.4)$ & $1(2.6)$ & $4(4.7)$ \\
T2 & $4(8.5)$ & $10(26.3)$ & $14(16.5)$ \\
T3 & $4(8.5)$ & $3(7.9)$ & $7(8.2)$ \\
T4 & $6(12.8)$ & $5(13.2)$ & $11(12.9)$ \\
Missing & $30(63.8)$ & $19(50.0)$ & $49(57.6)$ \\
Tumor Grade & & & \\
1 & $7(14.9)$ & $1(2.6)$ & $8(9.4)$ \\
2 & $8(17.0)$ & $11(28.9)$ & $19(22.3)$ \\
3 & $11(23.4)$ & $15(39.5)$ & $26(30.6)$ \\
Missing & $21(44.7)$ & $11(28.9)$ & $32(37.6)$ \\
Symptom Duration & & & \\
$<6$ months & $7(14.9)$ & $2(5.3)$ & $9(10.6)$ \\
$6-12$ months & $6(12.8)$ & $1(2.6)$ & $7(8.2)$ \\
$>12$ months & $6(12.8)$ & $8(21.0)$ & $14(16.5)$ \\
Missing & $28(59.6)$ & $27(71.0)$ & $55(64.7)$ \\
Lymph Node Status & & & \\
Involvement & $15(31.9)$ & $20(52.6)$ & $35(41.2)$ \\
No involvement & $4(8.5)$ & $3(7.9)$ & $7(8.2)$ \\
Missing & $28(59.6)$ & $15(39.5)$ & $43(50.6)$ \\
\hline Notes: T1 $\leq 20$ mm; T2 $>20 \mathrm{~mm}$ but $\leq 50 \mathrm{~mm} ;$ T3 $>50 \mathrm{~mm} ; \mathrm{T} 4 \mathrm{extension}$ to chest wall \\
or skin & & & \\
& & &
\end{tabular}

\section{Discussion}

Malawian women with breast cancer have long symptom durations prior to diagnosis, a majority have poorly differentiated tumors of ductal origin, and they are commonly diagnosed at young ages. These findings are consistent with other studies in the region where many patients are diagnosed with advanced disease and aggressive clinical features. Nearly half of cases had high-grade tumours and over $80 \%$ of those with available lymph node information had lymph node involvement. One potential explanation for this may be that women with more aggressive tumours seek care and receive diagnostic services compared to women with slower progressing tumours ${ }^{4}$. A majority of cases occurred in women under 50 years of age, and the mean age was 48.6 years. This is similar to findings from Blantyre, where the mean age of 19 cases from the Southern Region of Malawi was 50.7 years $^{11}$. It remains uncertain whether younger age at breast cancer diagnosis in African settings reflects environmental factors, genetic factors, differences in tumor biology, or the younger demographic profile of African countries overall. To our knowledge this is the first to attempt describe clinical features of breast cancer in Malawi, therefore this study provides essential baseline data regarding tumor biology and clinical presentations in our setting. We were unable to determine stage for most of the cancer cases due to limitations of available clinical records. We were unable to obtain detailed data regarding treatment and outcomes because patient charts were not accessible. However, these findings provide important insight into the local presentation of breast cancer. In particular, the absence of HR status testing in most African settings is a major deficiency in care that requires correction, not only in Malawi but throughout the region. HR positivity varies widely across Africa, ${ }^{4,12,13}$ and routine testing would ensure that women with HR-negative cancers are spared ineffective hormonal therapy. It would also identify many women who would benefit from tamoxifen, which is relatively inexpensive, well-tolerated, and available in Malawi. Detailed prospective studies of breast cancer in Malawi are urgently needed.

\section{Conclusions}

Aggressive breast cancer affects many young and middleaged Malawian women. There is a need for more expedient evaluation of breast masses to enable earlier diagnosis and treatment. Increasing breast cancer awareness is also imperative to address late diagnosis. Improved clinical and pathological characterization, including HR status, are needed to better understand this disease in our setting and improve outcomes. Such efforts are currently ongoing across Malawi and will hopefully lead to more detailed breast cancer data from Malawi moving forward.

\section{Acknowledgements}

We gratefully acknowledge the KCH Pathology Laboratory staff for their dedication, as well as $\mathrm{KCH}$ patients and clinicians. Ms. Kohler is supported by the UNC Hopkins Morehouse Tulane Fogarty Global Health Fellows Program (R25 TW009340) and by the UNC Cancer Care Quality Training Program (R25 CA116339). Professor Liomba is supported by the Medical Education Partnership Initiative (R24 TW008927-01). Dr. Gopal is supported by the Fogarty International Center (K01 TW009488), National Cancer Institute (R21 CA180815), AIDS Malignancy Consortium (U01 CA121947), and Program in Global Oncology of the UNC Lineberger Comprehensive Cancer Center (P30 CA016086).

\section{References}

1. Parkin DM, Bray F, Ferlay J, Jemal A. Cancer in Africa 2012. Cancer Epidemiology Biomarkers \& Prevention. 2014;23(6):953-966. 
2. Jemal A, Bray F, Center MM, Ferlay J, Ward E, Forman D. Global cancer statistics. CA: A Cancer Journal for Clinicians. 2011;61(2):6990.

3. Ly M, Antoine M, André F, Callard P, Bernaudin J-F, Diallo DA. Breast cancer in Sub-Saharan African women: review]. Bulletin du cancer. 2011;98(7):797.

4. Sayed S, Moloo Z, Wasike R, et al. Is breast cancer from Sub Saharan Africa truly receptor poor? Prevalence of ER/PR/HER2 in breast cancer from Kenya. The Breast. (0).

5. Adjei EK, Owusu-Afriyie O, Awuah B, Stalsberg H. Hormone Receptors and Her2 Expression in Breast Cancer in Sub-Saharan Africa. A Comparative Study of Biopsies from Ghana and Norway. The breast journal. 2014;20(3):308-311.

6. Adisa C, Eleweke N, Alfred A, et al. Biology of breast cancer in Nigerian women: A pilot study. Annals of African medicine. 2012;11(3):169.

7. Adesina A, Chumba D, Nelson AM, et al. Improvement of pathology in sub-Saharan Africa. The lancet oncology. 2013;14(4):e152-e157.

8. Gopal S, Krysiak R, Liomba NG, et al. Early Experience after Developing a Pathology Laboratory in Malawi, with Emphasis on Cancer Diagnoses. PloS one. 2013;8(8):e70361.
9. GLOBOCAN 2012 v1.0, Cancer Incidence and Mortality Worldwide: IARC CancerBase No. 11. 2013. http://globocan.iarc.fr. Accessed 01 April 2014.

10. Msyamboza KP, Dzamalala C, Mdokwe C, et al. Burden of cancer in Malawi; common types, incidence and trends: National populationbased cancer registry. BMC research notes. 2012;5(1):149.

11. Mtonga P, Masamba L, Milner D, Shulman L, Nyirenda R, Mwafulirwa K. Biopsy case mix and diagnostic yield at a Malawian central hospital. Malawi Medical Journal. 2013;25(3):62-64.

12. Ly M, Antoine M, Dembélé AK, et al. High Incidence of TripleNegative Tumors in Sub-Saharan Africa: A Prospective Study of Breast Cancer Characteristics and Risk Factors in Malian Women Seen in a Bamako University Hospital. Oncology. 2012;83(5):257-263.

13. McCormack VA, Joffe M, van den Berg E, et al. Breast cancer receptor status and stage at diagnosis in over 1,200 consecutive public hospital patients in Soweto, South Africa: a case series. Breast Cancer Research. 2013;15(5):R84. 